\title{
Predictive Utility of Changes in Optic Nerve Sheath Diameter after Cardiac Arrest for Neurologic Outcomes
}

\author{
Heekyung Lee ${ }^{1,2,+}$, Joonkee Lee ${ }^{1,2,+}$, Hyungoo Shin $1,3, * \mathbb{C}$, Changsun Kim ${ }^{1,3}$, Hyuk-Joong Choi ${ }^{1,3}$ \\ and Bo-Seung Kang ${ }^{1,3}$ \\ 1 Department of Emergency Medicine, Hanyang University Guri Hospital, 153, Gyeongchunro-ro, Guri, \\ Gyeonggi-do 11923, Korea; massdt@naver.com (H.L.); xjkx@naver.com (J.L.); flyes98@naver.com (C.K.); \\ airwaymanage@gmail.com (H.-J.C.); olivertw@hanyang.ac.kr (B.-S.K.) \\ 2 Department of Emergency Medicine, Graduate School of Medicine, Hanyang University, Seoul 04763, Korea \\ 3 Department of Emergency Medicine, College of Medicine, Hanyang University, 222, Wangsimni-ro, \\ Seongdong-gu, Seoul 04763, Korea \\ * Correspondence: seodtst@gmail.com; Tel.: +82-31-560-2058 \\ + These authors contributed equally to this work.
}

Citation: Lee, H.; Lee, J.; Shin, H.; Kim, C.; Choi, H.-J.; Kang, B.-S.

Predictive Utility of Changes in Optic Nerve Sheath Diameter after Cardiac Arrest for Neurologic Outcomes. Int. J. Environ. Res. Public Health 2021, 18, 6567. https://doi.org/10.3390/ ijerph18126567

Academic Editor: Je Hyeok Oh

Received: 5 May 2021

Accepted: 16 June 2021

Published: 18 June 2021

Publisher's Note: MDPI stays neutral with regard to jurisdictional claims in published maps and institutional affiliations.

Copyright: (c) 2021 by the authors. Licensee MDPI, Basel, Switzerland. This article is an open access article distributed under the terms and conditions of the Creative Commons Attribution (CC BY) license (https:/ / creativecommons.org/licenses/by/ $4.0 /)$.

\begin{abstract}
The optic nerve sheath diameter (ONSD) can help predict the neurologic outcomes of patients with post-cardiac arrest (CA) return of spontaneous circulation (ROSC). We aimed to investigate the effect of ONSD changes before and after CA on neurologic outcomes in patients with ROSC after CA using brain computed tomography (CT). The study included patients hospitalized after CA, who had undergone pre- and post-CA brain CT between January 2001 and September 2020. The patients were divided into good and poor neurologic outcome (GNO and PNO, respectively) groups based on their neurologic outcome at hospital discharge. We performed between-group comparisons of the amount and rate of ONSD changes in brain CT and calculated the area under the curve (AUC) to determine their predictive value for neurologic outcomes. Among the 96 enrolled patients, 25 had GNO. Compared with the GNO group, the PNO group showed a significantly higher amount ( 0.30 vs. $0.63 \mathrm{~mm} ; p=0.030)$ and rate (5.26 vs. $12.29 \% ; p=0.041)$ of change. The AUC for predicting PNO was $0.64(95 \%$ confidence interval $=0.53-0.73 ; p=0.04)$, and patients with a rate of ONSD change $>27.2 \%$ had PNO with $100 \%$ specificity and positive predictive value. Hence, ONSD changes may predict neurologic outcomes in patients with post-CA ROSC.
\end{abstract}

Keywords: heart arrest; optic nerve sheath diameter; patient outcome assessment

\section{Introduction}

Ischemia/reperfusion cerebral injury after cardiac arrest (CA) may cause cerebral edema [1,2]. This results in an increase in intracranial pressure (ICP) and contributes to poor neurologic outcomes in patients with post-CA return of spontaneous circulation (ROSC) [3,4]. In these survivors, there is a need for the early detection of increased ICP and prediction of neurologic outcomes to facilitate appropriate post-resuscitation care [5]. This can help prioritize the allocation of limited medical resources to patients with expected good neurologic outcomes. There have been studies on various predictive factors for post-CA neurologic outcomes, including neurologic examination of brainstem reflexes, electrophysiological tests, and serum biomarkers, such as neuron-specific enolase and S-100B [6-8]. However, these have been recommended as prognostic factors at $72 \mathrm{~h}$ postCA [8-10]. Moreover, early brain computed tomography (CT) of patients with post-CA ROSC may play a crucial role as a prognostic predictor. Further, the American Heart Association guidelines recommend early post-CA brain CT scans and confirm that a decrease in the gray-to-white matter ratio (GWR) can help predict neurologic outcomes [3,10-12]. Additionally, there have been studies regarding the role of the optic nerve sheath diameter (ONSD) on brain CT in predicting neurologic outcomes in post-CA survivors [13-15]. 
Previous studies have indicated the potential role of the ONSD on brain CT as a useful tool for non-invasive ICP measurement $[16,17]$. Additionally, recent studies have demonstrated that the ONSD on brain CT is useful for early neurologic outcome prediction through the evaluation of increased ICP in patients with post-CA ROSC $[14,18]$. Two recent meta-analyses confirmed the utility of the ONSD as a prognostic factor for neurologic outcomes in post-CA patients $[19,20]$. However, the methodology of these systematic reviews and meta-analyses is concerning $[19,20]$. The definition of poor neurologic outcome (PNO) included both Glasgow-Pittsburgh Cerebral Performance Categories (CPCs) 5 and CPC 3-5, and the outcome measurement time had a wide range (from hospital discharge to 6 months post-discharge). These limitations may have influenced the results of the studies. Furthermore, most of the studies included in the meta-analysis indicated that the sole use of the ONSD had limited predictive utility for prognosis. All these studies measured only the post-CA ONSD values without considering changes within individuals.

This study aimed to assess the differences between the pre- and post-CA ONSDs in patients with ROSC after CA using brain CT imaging. Additionally, we aimed to investigate the impact of the amount and rate of post-CA ONSD changes on the neurologic outcome at discharge.

\section{Materials and Methods}

\subsection{Study Design and Population}

This retrospective observational cohort study investigated brain CT scans of patients hospitalized after CA at a single university-affiliated hospital in Korea between January 2001 and September 2020. This study was approved by the Institutional Review Board of Hanyang University Guri Hospital (IRB No. GURI 2020-12-008). The requirement for informed consent was waived due to the retrospective nature of the study.

We included adult patients hospitalized after CA who underwent pre- and post-CA brain CT. The exclusion criteria were as follows: (1) transfer to another hospital after ROSC, (2) age <19 years, (3) traumatic/non-traumatic brain hemorrhage or brain tumor, (4) a history of ophthalmological disorders or surgeries that could affect the ONSD, and (5) the most recent pre-CA brain CT was performed at an age $<19$ years. Finally, eligible patients were divided into the good neurologic outcome (GNO) and poor neurologic outcome (PNO) groups based on their neurologic outcome at discharge; subsequently, we measured ONSD changes and performed between-group analysis. The primary outcome was the association between ONSD changes and the neurologic outcomes of patients hospitalized after CA.

\subsection{Data Collection}

We retrospectively collected the following data from electronic medical records: age; sex; comorbidities (hypertension, diabetes, and myocardial infarction); etiology (cardiac and respiratory); the location of the CA; whether the CA was witnessed; bystander cardiopulmonary resuscitation (CPR); the first monitored shockable rhythm; the CA duration, including the no-flow time (the time between CA and CPR initiation) and the low-flow time (the time between active CPR and ROSC); and administered targeted temperature management (TTM). Based on the medical records, we determined the interval between the latest pre-CA brain CT and ROSC (month), which was termed "CT to ROSC", and between ROSC and post-CA brain CT (min), which was termed "ROSC to CT". Additionally, we collected data regarding the neurologic outcomes at discharge using the Glasgow-Pittsburgh CPC. Based on the CPC scale, we defined GNO and PNO as a CPC of 1 or 2 and 3-5, respectively.

\subsection{ONSD Measurements Using Brain CT}

Brain CT scans were performed based on standard protocols using non-contrast $4 \mathrm{~mm}$ contiguous slices parallel to the orbital floor from the skull base to the vertex. The pre-CA and post-CA ONSDs were bilaterally measured at $3 \mathrm{~mm}$ behind the globe on brain CT 
using the picture archiving and communication system (PACS) ruler tool (PiView STAR, INFINITT, Seoul, Korea). Images were magnified at $450 \%$ and changed to the "mediastinum window (window width: 440; window level: 45) using the PACS tool. The ONSDs of the right and left eyes were averaged to obtain the mean value. All measurements were performed by emergency physicians blinded to the patient information, including the neurologic outcomes. Additionally, we calculated the amount and rate of ONSD change. We defined the amount of change as the difference between the pre-CA and post-CA ONSD. Moreover, the rate of ONSD change was calculated as follows:

$$
\text { Rate of ONSD change }=\left(\frac{\text { Post }- \text { CA ONSD }- \text { Pre }- \text { CA ONSD }}{\text { Pre }- \text { CA ONSD }}\right) \times 100
$$

We used the following CT equipment: SOMATOM Sensation 16, SOMATOM Definition DS, and SOMATOM Definition Edge (Siemens Healthcare, Erlangen, Germany). The following parameters were used: $120 \mathrm{kVp}, 250-500 \mathrm{mAs}$, and 4 to $4.5 \mathrm{~mm}$ slice thickness. All CT images were stored in the Digital Imaging and Communication in Medicine format in the PACS.

\subsection{Sample Size}

We calculated the sample size based on a pilot study of 33 participants using $G^{*}$ Power (3.1.9.6; Heinrich Heine University, Düsseldorf, Germany). The mean ONSD of patients with GNO and PNO were $4.75 \pm 1.45 \mathrm{~mm}$ and $5.63 \pm 1.85 \mathrm{~mm}$, respectively. The required sample size was calculated as 90 participants (effect size: 0.53; a-error: 0.05; power: 0.8 ); finally, considering a 10\% drop-out rate, 99 participants were required.

\subsection{Statistical Analysis}

Continuous and categorical variables were reported as the median with interquartile range (IQR) and number with percentages, respectively. Normally distributed variables were analyzed using the Mann-Whitney $U$ test and Wilcoxon rank sum test, while nonnormally distributed variables were analyzed using the Shapiro-Wilk test. The chi-square test or Fisher's exact test was used to analyze categorical variables. Statistical significance was set at $p<0.05$. Multivariable analysis with logistic regression was used to determine the risk factors for poor neurologic outcomes, with adjustment for confounding variables found to be significant in univariate analysis. Variables with $p<0.2$ in univariate analysis with the rate of ONSD change were included in the multivariable analysis. Further, the Hosmer-Lemeshow test was used to confirm the logistic model calibrations. The predictive performance of the main outcome was assessed using the area under the receiver operating characteristic curve ((ROC) AUC) with a sensitivity over 1 indicating- specificity. Results were obtained using the Youden Index and presented as a 95\% confidence interval (CI) of AUC with sensitivity, specificity, positive predictive value (PPV), and negative predictive value (NPV). ROC analysis was performed using MedCalc Statistical Software (version 17.2, MedCalc Software, Ostend, Belgium), while the other statistical analyses were performed using SPSS software (version 25.0, IBM, Armonk, NY, USA).

\section{Results}

\subsection{Baseline Characteristics}

Among 145 post-CA survivors who underwent brain CT before and after CA, 49 patients were excluded as follows: 40 patients who were transferred to another hospital, seven patients with intracranial or subarachnoid hemorrhage, one patient with a brain tumor, and one patient aged $\leq 18$ years. Finally, we enrolled 96 patients and allocated them to the GNO $(n=25,26.0 \%)$ or PNO group $(n=71,74.0 \%)$ (Figure 1$)$. 


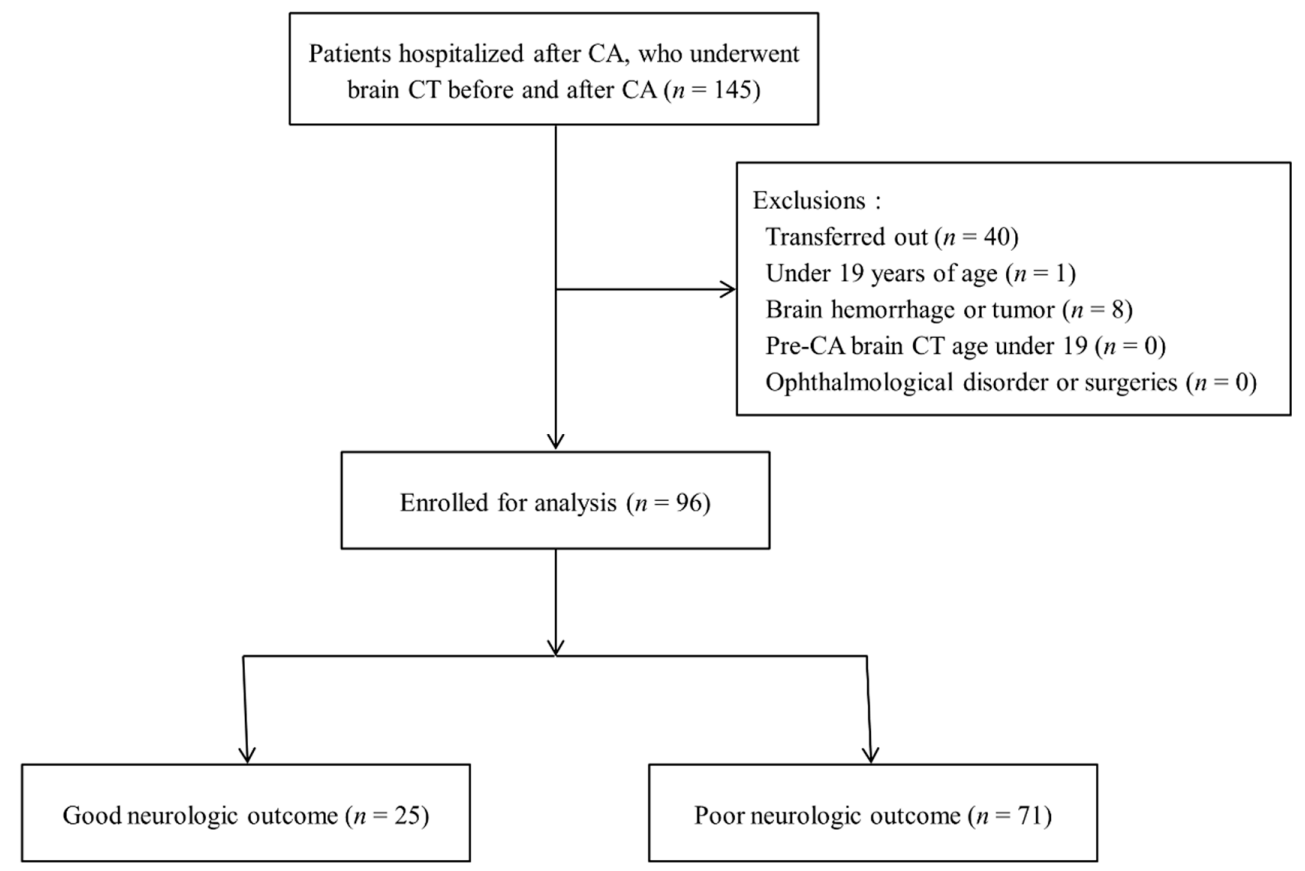

Figure 1. Flow chart of the study process.

Table 1 summarizes the patients' demographics and clinical characteristics. The median age of the included patients was 70 (IQR: 58-79) years, and $56.3 \%$ were male. The GNO group was significantly younger than the PNO group. Moreover, the GNO group displayed a significantly higher frequency of cardiac etiology and shockable rhythm, as well as shorter no-flow and low-flow times, than the PNO group. Contrastingly, out-of-hospital cardiac arrest was more frequent among the PNO group.

Table 1. Baseline characteristics of enrolled patients.

\begin{tabular}{|c|c|c|c|c|}
\hline & Total $(n=96)$ & GNO $(n=25)$ & PNO $(n=71)$ & $p$-Value \\
\hline \multicolumn{5}{|c|}{ Demographics } \\
\hline Age, year & $70(58-79)$ & $60(52-67)$ & $75(61-80)$ & $<0.001$ \\
\hline Sex, male & $54(56.3)$ & $15(60.0)$ & $39(54.9)$ & 0.660 \\
\hline \multicolumn{5}{|c|}{ Comorbidities } \\
\hline HTN & $52(54.2)$ & $14(56.0)$ & 38 (53.5) & 0.831 \\
\hline $\mathrm{DM}$ & 37 (38.5) & $6(24.0)$ & $31(43.7)$ & 0.082 \\
\hline MI & $16(16.7)$ & $4(16.0)$ & $12(16.9)$ & 1.000 \\
\hline \multicolumn{5}{|c|}{ Etiology } \\
\hline Cardiac & $23(24.0)$ & $14(56.0)$ & $9(12.7)$ & $<0.001$ \\
\hline Respiratory & 40 (41.7) & $8(32.0)$ & $32(45.1)$ & 0.254 \\
\hline Others & $33(34.4)$ & $3(12.0)$ & $30(42.3)$ & 0.006 \\
\hline \multicolumn{5}{|c|}{ Resuscitation } \\
\hline Location of arrest, OHCA & $76(79.2)$ & $16(64.0)$ & $60(84.5)$ & 0.030 \\
\hline Witnessed & $72(75.0)$ & $19(76.0)$ & $53(74.6)$ & 0.893 \\
\hline Bystander CPR & $61(63.5)$ & $18(72.0)$ & 43 (60.6) & 0.307 \\
\hline Shockable rhythm & 11 (11.5) & $8(32.0)$ & $3(4.2)$ & 0.001 \\
\hline No-flow time, $\min$ & $10(0-21)$ & $4(0-9)$ & $11(2-25)$ & 0.003 \\
\hline Low-flow time, $\mathrm{min}$ & $10(6-16)$ & $6(3-10)$ & $11(8-18)$ & 0.004 \\
\hline TTM & $6(6.3)$ & $3(12.0)$ & $3(4.2)$ & 0.180 \\
\hline CT to ROSC interval *, month & $27(6-55)$ & $40(6-55)$ & $23(6-53)$ & 0.780 \\
\hline ROSC to CT interval ${ }^{\dagger}, \min$ & $104(51-171)$ & $60(33-118)$ & $113(60-200)$ & 0.017 \\
\hline
\end{tabular}

Abbreviations: $\mathrm{GNO}=$ good neurologic outcome; $\mathrm{PNO}=$ poor neurologic outcome; $\mathrm{HTN}=$ hypertension; $\mathrm{DM}=\mathrm{di}-$ abetes mellitus; $\mathrm{MI}=$ myocardial infarction; $\mathrm{OHCA}=$ out-of-hospital cardiac arrest $; \mathrm{CPR}=$ cardiopulmonary resuscitation; TTM = targeted temperature management; $\mathrm{CT}=$ computed tomography; $\mathrm{ROSC}=$ return of spontaneous circulation. * The interval between the latest pre-CA brain CT and ROSC. ${ }^{+}$The interval between ROSC and post-CA brain CT. 


\subsection{Comparison of Pre-CA and Post-CA ONSDs}

In both groups, the post-CA ONSD was significantly higher than the pre-CA ONSD (Figure 2). The pre-CA ONSD and post-CA ONSD were 5.06 and $5.50 \mathrm{~mm}$, respectively, $(p<0.001)$ in the GNO group, and 5.07 and $5.72 \mathrm{~mm}$, respectively, $(p=0.001)$ in the PNO group (Supplemental Table S1).

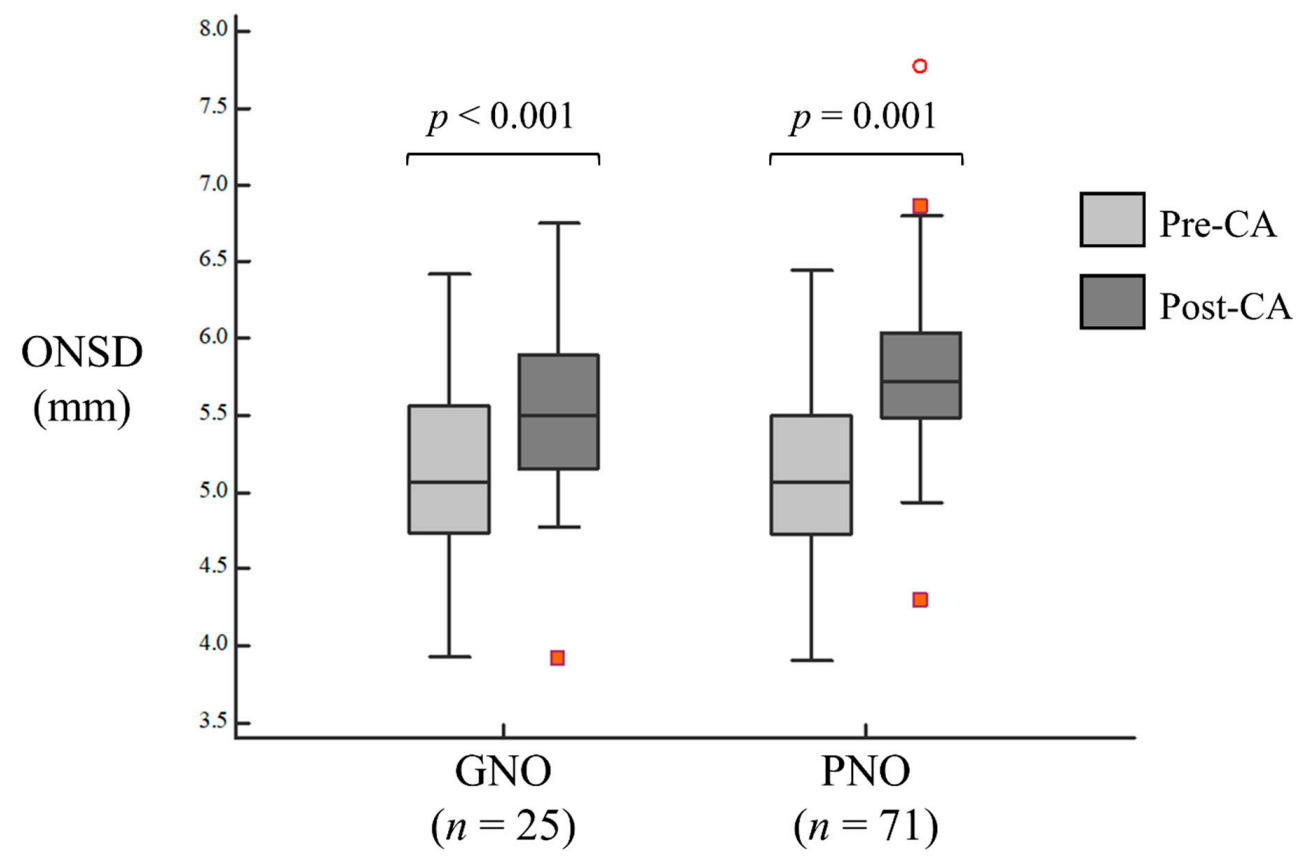

Figure 2. Comparison of the optic nerve sheath diameter between pre-cardiac arrest and post-cardiac arrest in good and poor neurologic outcome groups. The red circle and cubes mean outliers.

\subsection{The Association between ONSD Changes and Neurologic Outcomes}

Table 2 presents the between-group comparisons of the amount and rate of ONSD changes. There were no significant between-group differences in the pre-CA ONSD (5.06 vs. $5.07 \mathrm{~mm}, p=0.967$ ) and the post-CA ONSD (5.50 vs. $5.72 \mathrm{~mm}, p=0.075)$. However, the amount of ONSD change in the GNO group was significantly lower than that in the PNO group ( 0.30 vs. $0.63 \mathrm{~mm}, p=0.030)$. Additionally, the rate of ONSD change in the GNO group was significantly lower than that in the PNO group (5.26 vs. $12.29 \%, p=0.041$ ). Multivariable analysis revealed no independent association between the rate of ONSD change and poor neurologic outcome $(\mathrm{OR}=1.075 ; 95 \% \mathrm{CI}=0.990-1.167 ; p=0.084)$ (Table 3$)$.

Table 2. The comparisons of the amount and rate of ONSD changes between good and poor neurologic outcomes.

\begin{tabular}{|c|c|c|c|c|}
\hline & Total $(n=96)$ & GNO $(n=25)$ & PNO $(n=71)$ & $p$-Value \\
\hline \multicolumn{5}{|c|}{ Optic nerve sheath diameter } \\
\hline Pre-CA, mm & $5.07(4.73-5.52)$ & $5.06(4.76-5.53)$ & $5.07(4.73-5.52)$ & 0.967 \\
\hline Post-CA, mm & $5.66(5.41-6.01)$ & $5.50(5.16-5.88)$ & $5.72(5.49-6.04)$ & 0.075 \\
\hline \multicolumn{5}{|c|}{ Optic nerve sheath diameter changes between pre-CA and post-CA } \\
\hline Amount of change, $\mathrm{mm}$ & $0.57(0.25-0.84)$ & $0.30(0.18-0.65)$ & $0.63(0.32-0.87)$ & 0.030 \\
\hline Rate of change, $\%$ & $\begin{array}{c}11.10 \\
(4.70-17.21)\end{array}$ & $5.26(3.85-14.15)$ & $\begin{array}{c}12.29 \\
(5.83-18.74)\end{array}$ & 0.041 \\
\hline
\end{tabular}

Abbreviations: $\mathrm{GNO}$ = good neurologic outcome; $\mathrm{PNO}$ = poor neurologic outcome; $\mathrm{CA}$ = cardiac arrest. 
Table 3. Multivariable logistic regression analysis for poor neurologic outcome with baseline variables and rate of optic nerve sheath diameter change.

\begin{tabular}{ccc}
\hline Variables & Adjusted OR (95\% CI) & $p$-Value \\
\hline Age, year & $1.115(1.031-1.206)$ & 0.006 \\
DM & $3.358(0.636-17.733)$ & 0.154 \\
Shockable rhythm & $0.084(0.008-0.911)$ & 0.042 \\
No-flow time, min & $1.113(1.003-1.235)$ & 0.043 \\
Low-flow time, min & $1.123(1.024-1.231)$ & 0.013 \\
TTM & $0.119(0.008-1.794)$ & 0.124 \\
Location of arrest, OHCA & $0.833(0.115-6.014)$ & 0.856 \\
ROSC to CT interval *, min & $0.999(0.999-1.000)$ & 0.086 \\
Etiology, cardiac & $0.080(0.012-0.558)$ & 0.011 \\
Rate of change, \% & $1.075(0.990-1.167)$ & 0.084
\end{tabular}

Abbreviations: OR = odds ratio; DM = diabetes mellitus; TTM = targeted temperature management; OHCA = outof-hospital cardiac arrest; $\mathrm{ROSC}=$ return of spontaneous circulation; $\mathrm{CT}=$ computed tomography. ${ }^{*}$ The interval between ROSC and post-CA brain CT.

\subsection{Diagnostic Value of ONSD Changes for Predicting the Neurologic Outcome}

The AUC for predicting PNO was $0.64(95 \% \mathrm{CI}=0.53-0.73 ; p=0.04)$ in the ROC curve for the rate of ONSD change (Figure 3). Patients with a rate of ONSD change $>27.2 \%$ had PNO with a specificity and PPV of $100 \%$. GNO could be predicted using a cut-off value of $\leq 5.83 \%$ in the ROC curve for the rate of ONSD change, with a sensitivity and specificity of 60.0 and $76.06 \%$, respectively; the PPV and NPV were 46.9 and $84.4 \%$, respectively (Table 4).

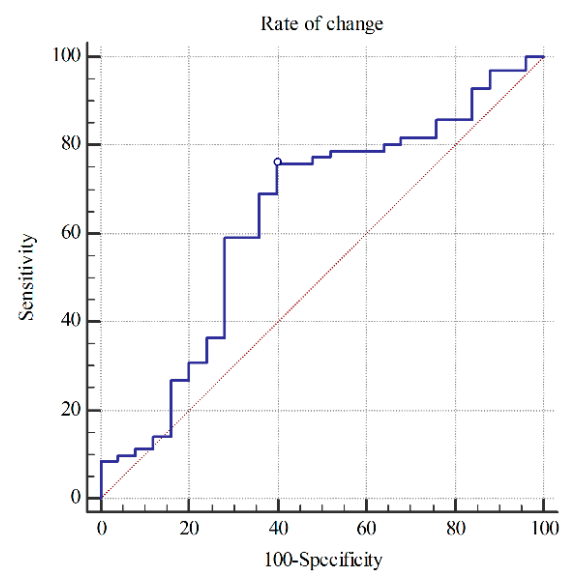

Figure 3. Receiver operator curve for predicting poor neurologic outcome using rate of optic nerve sheath diameter change. AUC $=0.64$ (95\% confidence interval $=0.53-0.73)$.

Table 4. Cut-off and diagnostic value of optic nerve sheath diameter change for predicting good and poor neurologic outcomes.

\begin{tabular}{cccccc}
\hline & Cut-Off, \% & Sensitivity & Specificity & PPV & NPV \\
\hline $\begin{array}{c}\text { Rate of change for } \\
\text { predicting PNO }\end{array}$ & $>27.2$ & 0.085 & 1.000 & 1.000 & 0.278 \\
$\begin{array}{c}\text { Rate of change for } \\
\text { predicting GNO }\end{array}$ & $\leq 5.83$ & 0.600 & 0.761 & 0.469 & 0.844
\end{tabular}

Abbreviations: PPV = positive predictive value; NPV = negative predictive value; $\mathrm{PNO}$ = poor neurologic outcome; $\mathrm{GNO}=$ good neurologic outcome.

\section{Discussion}

This study revealed that the amount and rate of ONSD change were significantly associated with neurologic outcomes. However, there was no significant between-group difference in the post-CA ONSD and no independent association of the rate of ONSD change with neurologic outcome after adjusting for confounding variables. Together 
with other established predictors, the rate of ONSD change may be useful for predicting neurologic outcomes. To the best of our knowledge, this is the first study to investigate individual differences in ONSD changes among post-CA survivors.

Previous studies have reported an association of neurologic outcomes among critically ill patients, including post-CA survivors, with increased ICP values [3,4,21,22]. The optic nerve sheath enclosing the optic nerve comprises a subarachnoid space layer, which is filled with cerebrospinal fluid (CSF) [23]; hence, ICP is positively correlated with the CSF pressure and the ONSD [23,24]. The ONSD is a potential non-invasive ICP estimator and could be useful for assessing intracranial hypertension [24]. In patients with post-CA hypoxic cerebral injury, increased ICP is associated with the neurologic outcome [3,21,22].

Several studies have reported that the ONSD can predict neurologic outcomes in postCA survivors. A retrospective cohort study from Korea reported an association between higher ONSD values on initial brain CT and poor neurologic outcomes [14]. Chelly et al. demonstrated the potential role of the ONSD as an early prediction tool for outcomes in post-CA patients treated with TTM administration [18]. Other studies have applied the ONSD in combination with other predictors, including GWR and albumin levels, to enhance the predictive value $[13,19,21]$. Moreover, two recent meta-analyses reported the potential use of the ONSD in predicting neurologic outcomes [19,20]. A registry-based multicenter study demonstrated inconsistency with these previous findings, as it reported no correlation between the ONSD on early unenhanced brain CT and neurologic outcomes in post-CA survivors managed with TTM administration [25]. Furthermore, previous studies indicated a limited and insufficient role of the post-CA ONSD alone in predicting neurologic outcomes in post-CA survivors [26].

The ONSD can provide non-invasive ICP measurement and could serve as a surrogate marker for increased ICP $[16,17]$. However, in healthy adults, there are differences in the baseline ONSD according to individual characteristics, including sex, body mass index (BMI), race, and eyeball size $[27,28]$. Most studies performed on healthy volunteers have reported that the mean ONSD ranges from approximately 3 to $5 \mathrm{~mm}$; furthermore, the reported mean or median ONSD values have varied across study cohorts, depending on race or measurement tools [27-30]. Ultrasonographic evaluation of healthy Asians revealed a higher ONSD value in males and individuals with a high BMI [27,28]. Therefore, these individual differences could confound the interpretation of the post-CA ONSD; furthermore, considering the baseline, ONSDs may help in improving the prognostic value. Thus, ONSD changes are potentially useful markers for ICP measurement changes. A prospective observational study on ONSD changes in patients with hydrocephalus reported a significant reduction in the ONSD after a ventriculoperitoneal shunt operation [31]. In our study, ONSD changes were more reliable than the ONSD itself in predicting neurologic outcomes in patients with post-CA.

A recent meta-analysis reported that, in comparison to $\mathrm{CT}$ and magnetic resonance imaging (MRI), sonographic measurement more accurately predicted neurologic outcomes among patients with post-CA [19]. However, obtaining and comparing pre-CA and postCA ONSDs using ultrasound has limitations in clinical settings. Moreover, determining the pre-CA ONSD using brain MRI also has limitations, given its specific modality. Recent studies indicate that the axial proton density/T2-weighted turbo spin-echo fat-suppressed sequence is required for ONSD measurements using MRI. However, in most post-CA patients, the turbo spin echo image is not included in the diffusion-weighted MRI [32,33]. Additionally, there is a strong association between the ONSD and eyeball transverse diameter (ETD) (ONSD/ETD ratio) in healthy adults [34]. However, there is a need for further studies on the association between the ONSD/ETD ratio and neurologic outcomes among post-CA patients.

This study has several limitations. First, this was a single-center study with a limited sample size that led to insufficient statistical power; however, we performed a power analysis to calculate the sample size, which was relatively large compared with those of other studies. Second, this retrospective study included patients who underwent both 
pre- and post-CA brain CT, which could lead to selection bias affecting the results. Third, although we attempted to extensively collect variables based on the Utstein Resuscitation Registry Templates, there may still be hidden confounders [35]. Fourth, there could have been minor measurement errors given the very small size of the ONSD in brain $\mathrm{CT}$. However, to minimize these errors, two blinded emergency physicians performed measurements using a standardized method showing consensus. Fifth, current guidelines recommend a neurologic outcome assessment at 3 months after discharge [10]. However, we measured the neurologic outcomes at discharge and did not determine the long-term outcomes. Sixth, OHCA and in-hospital cardiac arrest (IHCA) were both included and analyzed in this study, despite the differences in the characteristics and proportion of GNO and PNO. Follow-up studies that include only OHCA or IHCA patients may be required. This was a retrospective study, and the clinical utility of the predictive value for prognosis remains unclear. Hence, there is a need for large-scale prospective studies to confirm our findings.

\section{Conclusions}

The rate and amount of ONSD changes in brain CT were significantly associated with neurologic outcomes in patients with post-CA. ONSD changes may be useful to predict neurologic outcomes in patients with post-CA.

Supplementary Materials: The following are available online at https://www.mdpi.com/article/ 10.3390/ijerph18126567/s1, Table S1: Comparison of the optic nerve sheath diameter between precardiac arrest and post-cardiac arrest in good neurologic outcome and poor neurologic outcome groups.

Author Contributions: Conceptualization, H.S.; methodology, H.S. and H.L.; software, H.L. and J.L.; validation, C.K., H.-J.C., and B.-S.K.; formal analysis, H.S. and H.L.; investigation, H.S. and H.L.; data curation, C.K., H.-J.C., and B.-S.K.; writing-original draft preparation, H.L. and J.L.; writing-review and editing, H.S. All authors have read and agreed to the published version of the manuscript.

Funding: This research received no external funding.

Institutional Review Board Statement: This study was approved by the Institutional Review Board of Hanyang University Guri Hospital (IRB No. GURI 2020-12-008), which waived the requirement of informed consent.

Informed Consent Statement: This retrospective medical record review study waived the requirement of informed consent.

Data Availability Statement: The datasets used and analyzed during the current study are available from the corresponding author on reasonable request.

Acknowledgments: This work was supported by the research fund of Hanyang University (HY2020).

Conflicts of Interest: The authors declare no conflict of interest.
Abbreviations
ONSD optic nerve sheath diameter
CA cardiac arrest
CT computed tomography
GNO good neurologic outcome
PNO poor neurologic outcome
ICP intracranial pressure
ROSC return of spontaneous circulation
GWR gray-to-white matter ratio
CPR cardiopulmonary resuscitation
TTM targeted temperature management 


$\begin{array}{ll}\text { CPCs } & \text { Cerebral Performance Categories } \\ \text { PACS } & \text { picture archiving and communication system } \\ \text { IQR } & \text { interquartile range } \\ \text { AUC } & \text { area under curve } \\ \text { ROC } & \text { receiver operating characteristic } \\ \text { CI } & \text { confidence interval } \\ \text { PPV } & \text { positive predictive value } \\ \text { NPV } & \text { negative predictive value } \\ \text { CSF } & \text { cerebrospinal fluid } \\ \text { BMI } & \text { body mass index } \\ \text { ETD } & \text { eyeball transverse diameter }\end{array}$

\section{References}

1. Gueugniaud, P.Y.; Garcia-Darennes, F.; Gaussorgues, P.; Bancalari, G.; Petit, P.; Robert, D. Prognostic significance of early intracranial and cerebral perfusion pressures in post-cardiac arrest anoxic coma. Intensive Care Med. 1991, 17, 392-398. [CrossRef] [PubMed]

2. Sundgreen, C.; Larsen, F.S.; Herzog, T.M.; Knudsen, G.M.; Boesgaard, S.; Aldershvile, J. Autoregulation of cerebral blood flow in patients resuscitated from cardiac arrest. Stroke 2001, 32, 128-132. [CrossRef] [PubMed]

3. Metter, R.B.; Rittenberger, J.C.; Guyette, F.X.; Callaway, C.W. Association between a quantitative CT scan measure of brain edema and outcome after cardiac arrest. Resuscitation 2011, 82, 1180-1185. [CrossRef] [PubMed]

4. Iida, K.; Satoh, H.; Arita, K.; Nakahara, T.; Kurisu, K.; Ohtani, M. Delayed hyperemia causing intracranial hypertension after cardiopulmonary resuscitation. Crit. Care Med. 1997, 25, 971-976. [CrossRef] [PubMed]

5. Sandroni, C.; Cavallaro, F.; Callaway, C.W.; Sanna, T.; D’Arrigo, S.; Kuiper, M.; Della Marca, G.; Nolan, J.P. Predictors of poor neurological outcome in adult comatose survivors of cardiac arrest: A systematic review and meta-analysis. Part 1: Patients not treated with therapeutic hypothermia. Resuscitation 2013, 84, 1310-1323. [CrossRef]

6. Young, G.B. Clinical practice. Neurologic prognosis after cardiac arrest. N. Engl. J. Med. 2009, 361, 605-611. [CrossRef]

7. Cloostermans, M.C.; van Meulen, F.B.; Eertman, C.J.; Hom, H.W.; van Putten, M.J. Continuous electroencephalography monitoring for early prediction of neurological outcome in postanoxic patients after cardiac arrest: A prospective cohort study. Crit. Care Med. 2012, 40, 2867-2875. [CrossRef]

8. Sandroni, C.; Cariou, A.; Cavallaro, F.; Cronberg, T.; Friberg, H.; Hoedemaekers, C.; Horn, J.; Nolan, J.P.; Rossetti, A.O.; Soar, J. Prognostication in comatose survivors of cardiac arrest: An advisory statement from the European Resuscitation Council and the European Society of Intensive Care Medicine. Resuscitation 2014, 85, 1779-1789. [CrossRef]

9. Kamps, M.J.A.; Horn, J.; Oddo, M.; Fugate, J.E.; Storm, C.; Cronberg, T.; Wijman, C.A.; Wu, O.; Binnekade, J.M.; Hoedemaekers, C.W.E. Prognostication of neurologic outcome in cardiac arrest patients after mild therapeutic hypothermia: A meta-analysis of the current literature. Intensive Care Med. 2013, 39, 1671-1682. [CrossRef]

10. Geocadin, R.G.; Callaway, C.W.; Fink, E.L.; Golan, E.; Greer, D.M.; Ko, N.U.; Lang, E.; Licht, D.J.; Marino, B.S.; McNair, N.D.; et al. Standards for Studies of Neurological Prognostication in Comatose Survivors of Cardiac Arrest: A Scientific Statement from the American Heart Association. Circulation 2019, 140, e517-e542. [CrossRef] [PubMed]

11. Lee, Y.H.; Oh, Y.T.; Ahn, H.C.; Kim, H.S.; Han, S.J.; Lee, J.J.; Lee, T.H.; Seo, J.Y.; Shin, D.H.; Ha, S.O.; et al. The prognostic value of the grey-to-white matter ratio in cardiac arrest patients treated with extracorporeal membrane oxygenation. Resuscitation 2016, 99, 50-55. [CrossRef]

12. Lee, B.K.; Kim, W.Y.; Shin, J.; Oh, J.S.; Wee, J.H.; Cha, K.C.; Park, Y.; Choi, J.H.; Jeung, K.W.; Korean Hypothermia Network Investigators. Prognostic value of gray matter to white matter ratio in hypoxic and non-hypoxic cardiac arrest with non-cardiac etiology. Am. J. Emerg. Med. 2016, 34, 1583-1588. [CrossRef] [PubMed]

13. Chae, M.K.; Ko, E.; Lee, J.H.; Lee, T.R.; Yoon, H.; Hwang, S.Y.; Cha, W.C.; Shin, T.G.; Sim, M.S.; Jo, I.J.; et al. Better prognostic value with combined optic nerve sheath diameter and grey-to-white matter ratio on initial brain computed tomography in post-cardiac arrest patients. Resuscitation 2016, 104, 40-45. [CrossRef] [PubMed]

14. Kim, Y.H.; Lee, J.H.; Hong, C.K.; Cho, K.W.; Yeo, J.H.; Kang, M.J.; Kim, Y.W.; Lee, K.Y.; Kim, J.J.; Hwang, S.Y. Feasibility of optic nerve sheath diameter measured on initial brain computed tomography as an early neurologic outcome predictor after cardiac arrest. Acad. Emerg. Med. 2014, 21, 1121-1128.

15. Ryu, J.-A.; Chung, C.R.; Cho, Y.H.; Sung, K.; Suh, G.Y.; Park, T.K.; Bin Song, Y.; Hahn, J.-Y.; Choi, J.-H.; Gwon, H.-C.; et al. The association of findings on brain computed tomography with neurologic outcomes following extracorporeal cardiopulmonary resuscitation. Crit Care 2017, 21, 15. [CrossRef]

16. Sekhon, M.S.; Griesdale, D.E.; Robba, C.; McGlashan, N.; Needham, E.; Walland, K.; Shook, A.C.; Smielewski, P.; Czosnyka, M.; Gupta, A.K.; et al. Optic nerve sheath diameter on computed tomography is correlated with simultaneously measured intracranial pressure in patients with severe traumatic brain injury. Intensive Care Med. 2014, 40, 1267-1274. [CrossRef]

17. Rajajee, V.; Fletcher, J.J.; Rochlen, L.R.; Jacobs, T.L. Comparison of accuracy of optic nerve ultrasound for the detection of intracranial hypertension in the setting of acutely fluctuating vs stable intracranial pressure: Post-hoc analysis of data from a prospective, blinded single center study. Crit Care 2012, 16, R79. [CrossRef] [PubMed] 
18. Chelly, J.; Deye, N.; Guichard, J.-P.; Vodovar, D.; Vong, L.; Jochmans, S.; Thieulot-Rolin, N.; Sy, O.; Serbource-Goguel, J.; Vinsonneau, C.; et al. The optic nerve sheath diameter as a useful tool for early prediction of outcome after cardiac arrest: A prospective pilot study. Resuscitation 2016, 103, 7-13. [CrossRef] [PubMed]

19. Lee, S.H.; Jong Yun, S. Diagnostic performance of optic nerve sheath diameter for predicting neurologic outcome in post-cardiac arrest patients: A systematic review and meta-analysis. Resuscitation 2019, 138, 59-67. [CrossRef] [PubMed]

20. Zhang, Y.W.; Zhang, S.; Gao, H.; Li, C.; Zhang, M.X. Prognostic Role of Optic Nerve Sheath Diameter for Neurological Outcomes in Post-Cardiac Arrest Patients: A Systematic Review and Meta-Analysis. BioMed Res. Int. 2020, 5219367.

21. You, Y.; Park, J.; Min, J.; Yoo, I.; Jeong, W.; Cho, Y.; Ryu, S.; Lee, J.; Kim, S.; Cho, S.; et al. Relationship between time related serum albumin concentration, optic nerve sheath diameter, cerebrospinal fluid pressure, and neurological prognosis in cardiac arrest survivors. Resuscitation 2018, 131, 42-47. [CrossRef] [PubMed]

22. Park, J.S.; You, Y.; Min, J.H.; Yoo, I.; Jeong, W.; Cho, Y.; Ryu, S.; Lee, J.; Kim, S.W.; Cho, S.U.; et al. Study on the timing of severe blood-brain barrier disruption using cerebrospinal fluid-serum albumin quotient in post cardiac arrest patients treated with targeted temperature management. Resuscitation 2018, 135, 118-123. [CrossRef] [PubMed]

23. Selhorst, J.B.; Chen, Y. The optic nerve. Semin. Neurol. 2009, 29, 29-35. [CrossRef] [PubMed]

24. Robba, C.; Santori, G.; Czosnyka, M.; Corradi, F.; Bragazzi, N.; Padayachy, L.; Taccone, F.S.; Citerio, G. Optic nerve sheath diameter measured sonographically as non-invasive estimator of intracranial pressure: A systematic review and meta-analysis. Intensive Care Med. 2018, 44, 1284-1294. [CrossRef] [PubMed]

25. Lee, D.H.; Lee, S.H.; Oh, J.H.; Cho, I.S.; Lee, Y.H.; Han, C.; Choi, W.J.; Sohn, Y.D.; KORHN Investigators. Optic nerve sheath diameter measured using early unenhanced brain computed tomography shows no correlation with neurological outcomes in patients undergoing targeted temperature management after cardiac arrest. Resuscitation 2018, 128, 144-150. [CrossRef]

26. Rush, B.; Wormsbecker, A.; Berger, L.; Wiskar, K.; Sekhon, M.S.; Griesdale, D.E. Optic nerve sheath diameter on computed tomography not predictive of neurological status post-cardiac arrest. CJEM 2017, 19, 181-185. [CrossRef] [PubMed]

27. Kim, D.H.; Jun, J.S.; Kim, R. Ultrasonographic measurement of the optic nerve sheath diameter and its association with eyeball transverse diameter in 585 healthy volunteers. Sci. Rep. 2017, 7, 15906. [CrossRef] [PubMed]

28. Wang, L.; Feng, L.; Yao, Y.; Deng, F.; Wang, Y.; Feng, J.; Xing, Y. Ultrasonographic Evaluation of Optic Nerve Sheath Diameter among Healthy Chinese Adults. Ultrasound Med. Biol. 2016, 42, 683-688. [CrossRef] [PubMed]

29. Maude, R.R.; Hossain, M.A.; Hassan, M.U.; Osbourne, S.; Sayeed, K.L.A.; Karim, M.R.; Samad, R.; Borooah, S.; Dhillon, B.; Day, N.P.J.; et al. Transorbital sonographic evaluation of normal optic nerve sheath diameter in healthy volunteers in Bangladesh. PLoS ONE 2013, 8, e81013. [CrossRef] [PubMed]

30. Romagnuolo, L.; Tayal, V.; Tomaszewski, C.; Saunders, T.; Norton, H.J. Optic nerve sheath diameter does not change with patient position. Am. J. Emerg. Med. 2005, 23, 686-688. [CrossRef] [PubMed]

31. Bhandari, D.; Udupi Bidkar, P.; Adinarayanan, S.; Narmadhalakshmi, K.; Srinivasan, S. Measurement of changes in optic nerve sheath diameter using ultrasound and computed tomography scan before and after the ventriculoperitoneal shunt surgery in patients with hydrocephalus-A prospective observational trial. Br. J. Neurosurg. 2019, 33, 125-130. [CrossRef]

32. Kang, C.; Min, J.H.; Park, J.S.; Lee, B.; Lee, D.; Chae, M.K. Relationship between optic nerve sheath diameter measured by magnetic resonance imaging, intracranial pressure, and neurological outcome in cardiac arrest survivors who underwent targeted temperature management. Resuscitation 2019, 145, 43-49. [CrossRef] [PubMed]

33. Geeraerts, T.; Newcombe, V.F.; Coles, J.P.; Abate, M.G.; Perkes, I.E.; Hutchinson, P.J.A.; Outtrim, J.G.; Chatfield, D.A.; Menon, D.K. Use of T2-weighted magnetic resonance imaging of the optic nerve sheath to detect raised intracranial pressure. Crit. Care 2008, 12, R114. [CrossRef] [PubMed]

34. Kim, D.H.; Jun, J.S.; Kim, R. Measurement of the optic nerve sheath diameter with magnetic resonance imaging and its association with eyeball diameter in healthy adults. J. Clin. Neurol. 2018, 14, 345-350. [CrossRef] [PubMed]

35. Perkins, G.D.; Jacobs, I.G.; Nadkarni, V.M.; Berg, R.A.; Bhanji, F.; Biarent, D.; Bossaert, L.L.; Brett, S.J.; Chamberlain, D.; de Caen, A.R.; et al. Cardiac arrest and cardiopulmonary resuscitation outcome reports: Update of the Utstein resuscitation registry templates for out-of-hospital cardiac arrest: A statement for healthcare professionals from a task force of the international liaison committee. Circulation 2015, 132, 1286-1300. [CrossRef] [PubMed] 